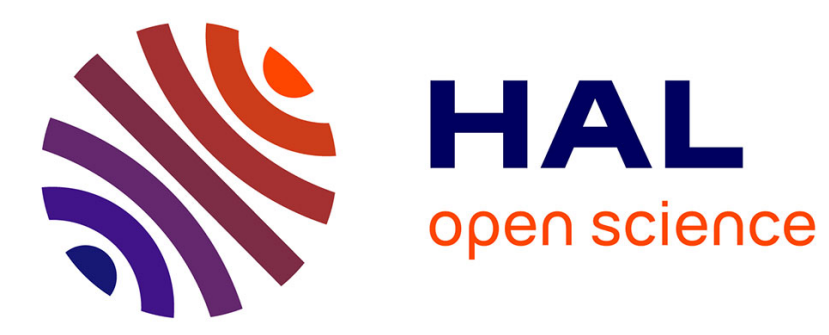

\title{
Simulation de la mise en forme d'un renfort déséquilibré
}

Damien Soulat, Jean-Luc Daniel, Philippe Boisse

\section{To cite this version:}

Damien Soulat, Jean-Luc Daniel, Philippe Boisse. Simulation de la mise en forme d'un renfort déséquilibré. Revue Européenne des Éléments Finis, 2004, 13 (5-7), pp.751-762. 10.3166/reef.13.751762 . hal-00020516

\section{HAL Id: hal-00020516 https://hal.science/hal-00020516}

Submitted on 10 Apr 2018

HAL is a multi-disciplinary open access archive for the deposit and dissemination of scientific research documents, whether they are published or not. The documents may come from teaching and research institutions in France or abroad, or from public or private research centers.
L'archive ouverte pluridisciplinaire HAL, est destinée au dépôt et à la diffusion de documents scientifiques de niveau recherche, publiés ou non, émanant des établissements d'enseignement et de recherche français ou étrangers, des laboratoires publics ou privés. 


\title{
Simulation de la mise en forme d'un renfort déséquilibré
}

\section{Damien Soulat* — Jean-Luc Daniel* — Philippe Boisse ***}

\author{
* Laboratoire de Mécanique des Systèmes et des Procédés \\ CNRS/ENSAM - Paris/Université d'Orléans \\ 8 rue Léonard de Vinci, F-45072 Orléans cédex \\ \{damien.soulat, jean-luc.daniel,philippe.boisse\}@univ-orleans.fr \\ **Laboratoire de Mécanique des Contacts et des Solides \\ CNRS/INSA-Lyon - Batiment Jacquard \\ 27avenue Jean Capelle, F-69621 Villeurbanne cédex \\ philippe.boisse@insa-lyon.fr
}

\begin{abstract}
RÉSUMÉ. La simulation de la mise en forme d'un renfort tissé très déséquilibré est décrite et comparée avec les essais expérimentaux. La simulation est réalisée grâce au développement d'éléments finis spécifiques aux tissus basés sur la seule énergie de déformation en tension. Ces éléments implémentés en dynamique explicite conduisent à une forme très simple des efforts intérieurs. Les résultats de la simulation traduisent correctement la forme très déséquilibrée constatée expérimentalement et due à la très forte différence de comportement dans les deux directions du renfort. Cet exemple montre la nécessité de prendre en compte les caractéristiques du comportement mécanique dans la simulation de la mise en forme, contrairement aux approches géométriques de type drapage.
\end{abstract}

ABSTRACT. The simulation of forming of a very unbalanced woven reinforcement is described and compared with the experimental tests. Simulation is carried out thanks to the development of finite elements specific to fabrics based on the only deformation energy in tension. These elements implemented in explicit dynamics lead to a very simple form of the interior efforts. The results of simulation correctly translate the shape very unbalanced noted in experiments and which had with the very strong difference in behavior in the two directions of the reinforcement. This example shows the need for taking into account the characteristics of the mechanical behavior in the simulation of forming, contrary to the geometrical approaches of draping type.

MOTS-CLÉS: mise en forme, simulation éléments finis, renforts tissés déséquilibrés, comportement mécanique des tissés.

KEYWORDS: experimental fabric forming, finite element simulations, unbalanced fabric, fabric mechanical behaviour. 


\section{Introduction}

L'utilisation de renforts tissés est en constante augmentation dans les industries aéronautiques et automobiles puisqu'ils permettent d'obtenir des structures composites fines, légères et de formes complexes en une seule opération. Le procédé RTM consiste à mettre en forme le renfort tissé sur lequel une résine est injectée (Carronier et al., 1996), (Bickerton et al., 1997). Lors de cette première phase de mise en forme les modes de déformations des tissus sont spécifiques au matériau utilisé autant qu'à sa constitution. Les codes de simulations sont nécessaires pour répondre d'une part, sur la faisabilité de la mise en forme et, d'autre part, pour prédire l'orientation des renforts qui va influer sur le comportement mécanique de la structure composite. De nombreux codes basés sur une approche géométrique ont été développés (algorithmes du filet, drapage) (Wang et al., 1999 ; Van der Ween, 1991 ; Long et al., 1994). Ces méthodes rapides et efficaces permettent de calculer les angles entre les mèches afin de les comparer à des valeurs limites pour lesquelles la mise en forme n'est plus possible sous peine de déchirures. Par contre, lors de mise en forme à l'aide d'un couple poinçonmatrice ces approches géométriques ne permettent pas de prendre en compte les conditions aux limites statiques (efforts sur ces outils) qui influent sur la forme obtenue. De la même manière les spécificités du matériau utilisé dans les mèches doivent être prises en compte dans l'analyse du procédé. La finalité de ce papier est de montrer sur l'exemple de la mise en forme d'un tissu très déséquilibré, les potentialités d'une méthode de simulation basée sur une approche mécanique à l'aide d'éléments finis spécifiques (Boisse et al., 2001). Le comportement spécifique de ce tissu ainsi que les essais réalisés à l'Université de Nottingham seront tout d'abord rappelés. Les éléments finis spécifiques proposés pour la modélisation par éléments finis sont constitués de mailles élémentaires de tissés, l'énergie de déformation élémentaire est calculée comme la somme des énergies de déformations de chacune de ces mailles. Cette approche est en bonne adéquation avec la physique des matériaux tissés puisqu'elle permet d'introduire le comportement suite aux résultats d'essais expérimentaux biaxiaux (Hivet et al., 2002). L'implémentation de ces éléments dans un logiciel à formulation explicite permettra de montrer la bonne corrélation avec les résultats des essais, et l'influence du comportement très déséquilibré sur la mise en forme.

\section{Essais expérimentaux de mise en forme}

Les essais ont été réalisés dans le laboratoire des composites de l'Université de Nottingham (Rudd et al., 1999). Le détail complet du procédé expérimental et des caractéristiques du renfort sont décrits dans (Dumont, 2003) La mise en forme est réalisée par un couple d'outils poinçon-matrice de forme hémisphérique monté sur une machine Instron de $25 \mathrm{KN}$ (figure 1). Pour limiter les plis, un anneau de $6 \mathrm{~kg}$ fait office de serre-flan. Les variations angulaires entre les mèches étant le mode 
principal de déformation lors de la mise en forme des tissus secs, une grille d'éléments initialement carrée a été dessinée sur le tissu avant la mise en forme afin de mesurer l'évolution de ces variations angulaires ainsi que les déformations dans chaque direction.

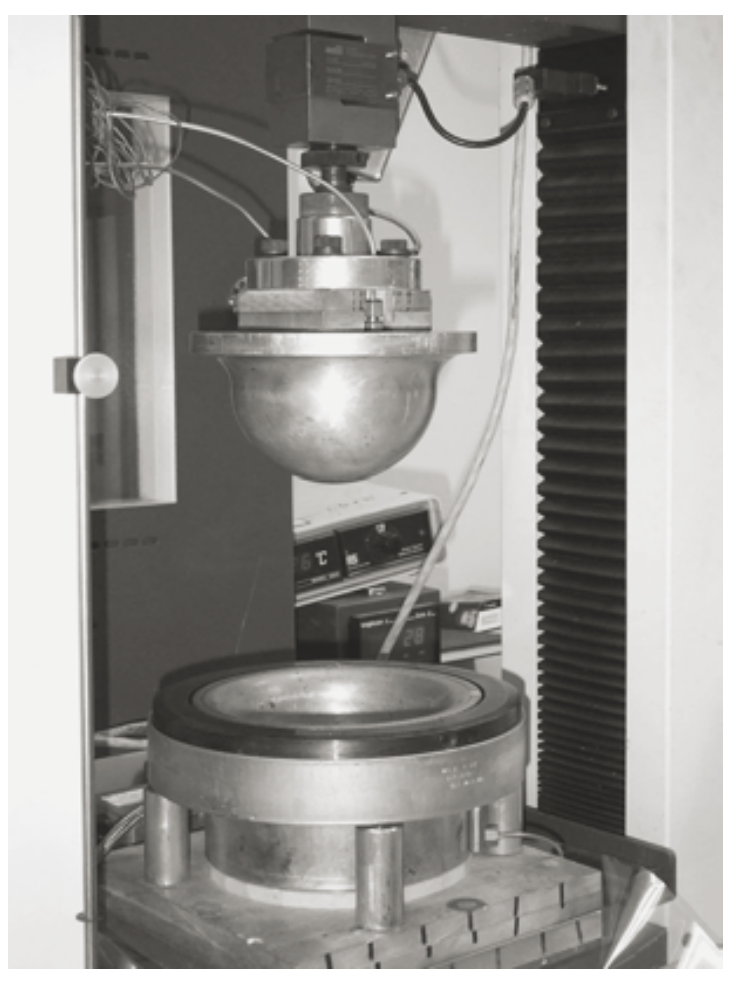

Figure 1. Couple poinçon-matrice

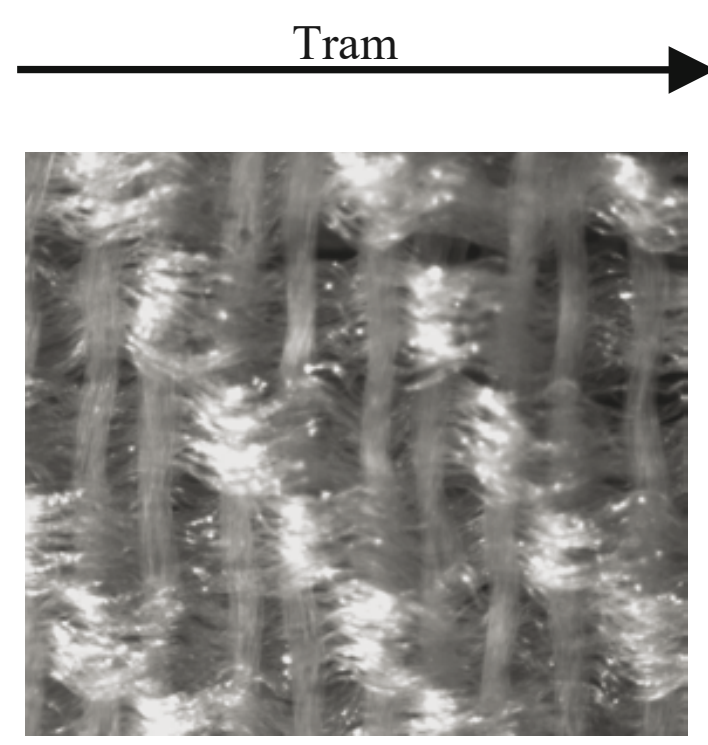

Figure 2. Tissage du renfort étudié

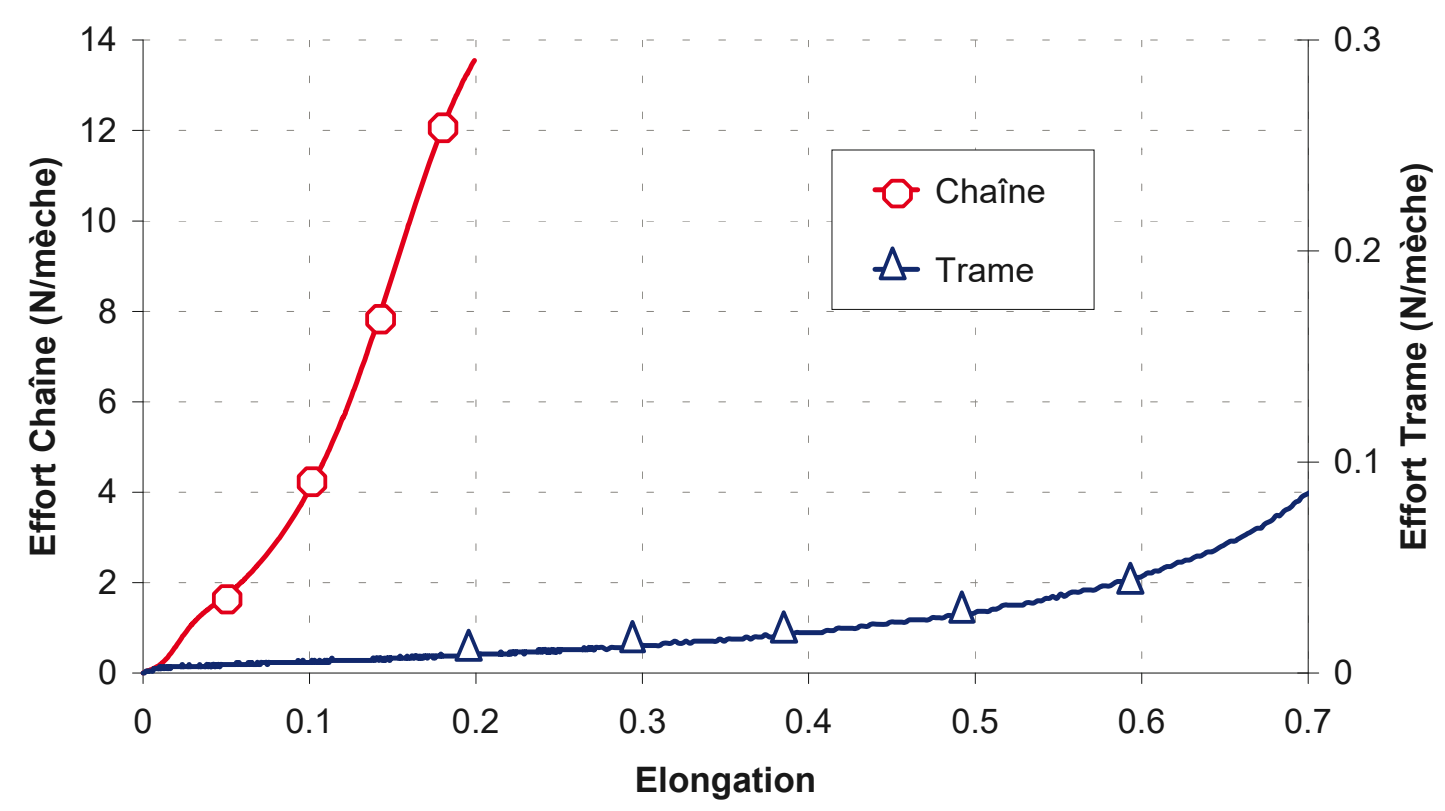

Figure 3. Comportement mécanique en tension dans chaque direction

Le tissu étudié est utilisé dans l'industrie automobile comme renfort de composites à matrice élastomère. La particularité de ce tissu réside dans les mèches 
de la direction trame qui sont très ondulées (figure 2). Les différences importantes de constitution entre les mèches dans les sens chaîne et trame conduisent à un comportement mécanique très déséquilibré (figure 3). Les tests de traction uniaxiaux réalisés au LMSP (Dumont, 2003) montrent par ailleurs un comportement non-linéaire. Pour un rang donné d'élongation, il est possible de définir un module d'Young équivalent dans chaque direction (tableau 1).

\begin{tabular}{|c|c|c|}
\hline $\begin{array}{c}\mathbf{E}_{\text {Chaine }}(\mathbf{N} / \text { mèche }) \\
\text { (jusqu'à rupture })\end{array}$ & $\begin{array}{c}\mathbf{E}_{\text {trame }}(\mathbf{N} / \text { mèche }) \\
(0<\text { Elongation }<0.85)\end{array}$ & $\mathbf{E}_{\text {chaîne }} / \mathbf{E}_{\text {trame }}$ \\
\hline 50 & 0.2 & 250 \\
\hline
\end{tabular}

Tableau 1. Module d'Young équivalents

\section{Simulation numérique de la mise en forme}

\subsection{Equation simplifiée du mouvement}

Les renforts de composite sont constitués de fibres qui sont elles-mêmes tissées sous forme de mèches. Cette structure interne autorise un mouvement possible entre les fibres. Par conséquent la plupart des rigidités sont très faibles comparativement à la rigidité en tension. Ces mouvements des mèches (ou ces rigidités nulles), qui sont un avantage lors de la mise en forme puisqu'ils permettent l'emboutissabilité du renfort, ne sont plus acceptables lors de l'utilisation de la pièce composite. Le rôle essentiel de la résine après l'injection sur la préforme est d'éviter ces mouvements des renforts. Par contre, pendant l'étape de mise en forme du tissu "sec" l'état de contrainte peut être défini de la manière suivante :

$$
\sigma=\sigma^{11} \mathbf{h}_{1} \otimes \mathbf{h}_{1}+\sigma^{22} \mathbf{h}_{2} \otimes \mathbf{h}_{2}
$$

où $\sigma$ désigne le tenseur des contraintes de Cauchy, $\mathbf{h}_{1}$ et $\mathbf{h}_{2}$ les vecteurs unitaires dans les directions chaîne et trame. Le teneur des tensions $\mathrm{T}$ est introduit par :

$$
\begin{aligned}
& \mathbf{T}=\mathrm{T}^{11} \mathbf{h}_{1} \otimes \mathbf{h}_{1}+\mathrm{T}^{22} \mathbf{h}_{2} \otimes \mathbf{h}_{2} \\
& \mathrm{~T}^{11}=\int_{\mathrm{A}_{1}} \sigma^{11} \mathrm{dS} \quad, \mathrm{T}^{22}=\int_{\mathrm{A}_{2}} \sigma^{22} \mathrm{dS} \quad, \mathrm{T}^{11} \geq 0, \mathrm{~T}^{22} \geq 0
\end{aligned}
$$

Pour un domaine constitué de "ncell" cellules élémentaires tissées, l'équation du mouvement peut s'écrire de la manière suivante (Boisse et al., 2001) : 


$$
\sum_{\mathrm{p}=1}^{\mathrm{ncelll}}{ }^{\mathrm{p}} \varepsilon_{11}(\eta){ }^{\mathrm{p}} \mathrm{T}^{11}{ }^{\mathrm{p}} \mathrm{L}_{1}+{ }^{\mathrm{p}} \varepsilon_{22}(\eta){ }^{\mathrm{p}} \mathrm{T}^{22}{ }^{\mathrm{p}} \mathrm{L}_{2}-\mathrm{T}_{\text {ext }}(\eta)=\int_{\Omega} \rho \ddot{\mathrm{u}} \eta \mathrm{dV}
$$

$\forall \eta / \eta=0 \quad \Gamma_{\mathrm{u}}$

$\boldsymbol{\eta}$ désigne le champ de déplacement virtuel tel que $\boldsymbol{\eta}=0$ sur le bord $\Gamma_{\mathrm{u}}$ du domaine

$\Omega . \mathrm{L}_{\alpha}(\alpha=1$ ou 2$)$ est la longueur de la cellule élémentaire dans la direction $\alpha . \varepsilon=\varepsilon_{\alpha \beta} \mathbf{h}^{\alpha} \otimes \mathbf{h}^{\beta} \quad$ est le tenseur du gradient symétrisé, $\rho$ est la masse volumique. u, $\dot{\mathbf{u}}, \ddot{\mathbf{u}}$ désignent les vecteurs déplacements, vitesses et accélérations.

f et $\mathbf{t}$ sont les efforts extérieurs de volume et sur le bord. Le travail virtuel des efforts extérieurs, Text $(\eta)$ est défini par :

$$
\mathrm{T}_{\text {ext }}(\eta)=\int_{\Omega} \mathrm{f} \eta \mathrm{dV}+\int_{\Gamma_{\mathrm{t}}} \mathrm{t} \eta \mathrm{dS}
$$

\subsection{Discrétisation par éléments finis en dynamique explicite}

L'équation du mouvement simplifiée [4] est utilisée dans une formulation discrétisée par éléments finis en dynamique explicite (la formulation implicite peutêtre trouvée dans (Boisse et al., 1997)) ; ainsi si le domaine d'étude $\Omega$ est divisé en éléments finis, l'équation [4] discrétisée est alors équivalente à :

$$
\mathbf{M} \ddot{\mathbf{u}}=\mathbf{F}_{\mathrm{ext}}-\mathbf{F}_{\mathrm{int}}
$$

où $\mathbf{M}$ désigne la matrice de masse, et Fext, Fint sont respectivement les vecteurs des efforts extérieurs et intérieurs. L'indice « $\mathrm{n}$ » se réfère aux quantités nodales, alors que l'exposant «e » désignent les quantités élémentaires. Dans le schéma aux différences centrales, la solution $\mathbf{u}_{\mathrm{n}}^{\mathrm{i}+1}$, au pas de temps $\Delta \mathrm{t}^{\mathrm{i}}$, entre les temps $\mathrm{t}^{\mathrm{i}}$ et $\mathrm{t}^{\mathrm{i}+1}$ est calculée à partir de $\mathbf{u}_{\mathrm{n}}^{\mathrm{i}}$ par la formule suivante :

$$
\mathbf{u}_{\mathrm{n}}^{\mathrm{i}+1}=\mathbf{u}_{\mathrm{n}}^{\mathrm{i}}+\left(\dot{\mathbf{u}}_{\mathrm{n}}^{\mathrm{i}-1 / 2}+\frac{1}{2}\left(\Delta \mathrm{t}^{\mathrm{i}-1}+\Delta \mathrm{t}^{\mathrm{i}}\right) \mathbf{M}_{\mathrm{D}}^{-1}\left(\mathbf{F}_{\text {ext }}^{\mathrm{i}}-\mathbf{F}_{\mathrm{int}}^{\mathrm{i}}\right)\right) \Delta \mathrm{t}^{\mathrm{i}}
$$

$\mathbf{M}_{\mathbf{D}}$ est une matrice diagonale issue de la matrice de masse (Zienkiewicz et al., 2001). Ce schéma est stable si et seulement si le pas de temps satisfait les conditions de stabilité (Belytschko, 1983). A partir de l'équation simplifiée [4] une forme spécifique aux tissés du travail virtuel des efforts intérieurs (et donc du vecteur des efforts intérieurs) est obtenue dans l'équation discrète [6] sous l'expression suivante :

$$
\mathrm{T}_{\text {int }}(\eta)=\sum_{\text {elt }} \sum_{\mathrm{p}=1}^{\text {ncell }}\left(\eta_{\mathrm{n}}{ }^{\mathrm{e}}\right)^{\mathrm{T}} \mathbf{F}_{\text {int }}^{\mathrm{e}}=\sum_{\text {elt }} \sum_{\mathrm{p}=1}^{\text {ncell }}{ }^{\mathrm{p}} \mathcal{E}_{11}(\eta)^{\mathrm{p}} \mathrm{T}^{11}{ }^{\mathrm{p}} \mathrm{L}_{1}+{ }^{\mathrm{p}} \mathcal{E}_{22}(\eta)^{\mathrm{p}} \mathrm{T}^{22}{ }^{\mathrm{p}} \mathrm{L}_{2}
$$


Et $\mathbf{F}_{\text {int }}=\underset{\text { elt }}{\mathrm{A}} \mathbf{F}_{\text {int }}^{\mathrm{e}}$, où $\mathrm{elt}_{\text {elt }}$ désigne l'opérateur d'assemblage sur les éléments.

\subsection{Efforts intérieurs pour un élément bilinéaire}

L'élément à 4 nœuds présenté à la figure 4 est composé de mèches. Le nombre de mèches respectivement dans le sens chaîne et trame est désigné par Nc et Nt.

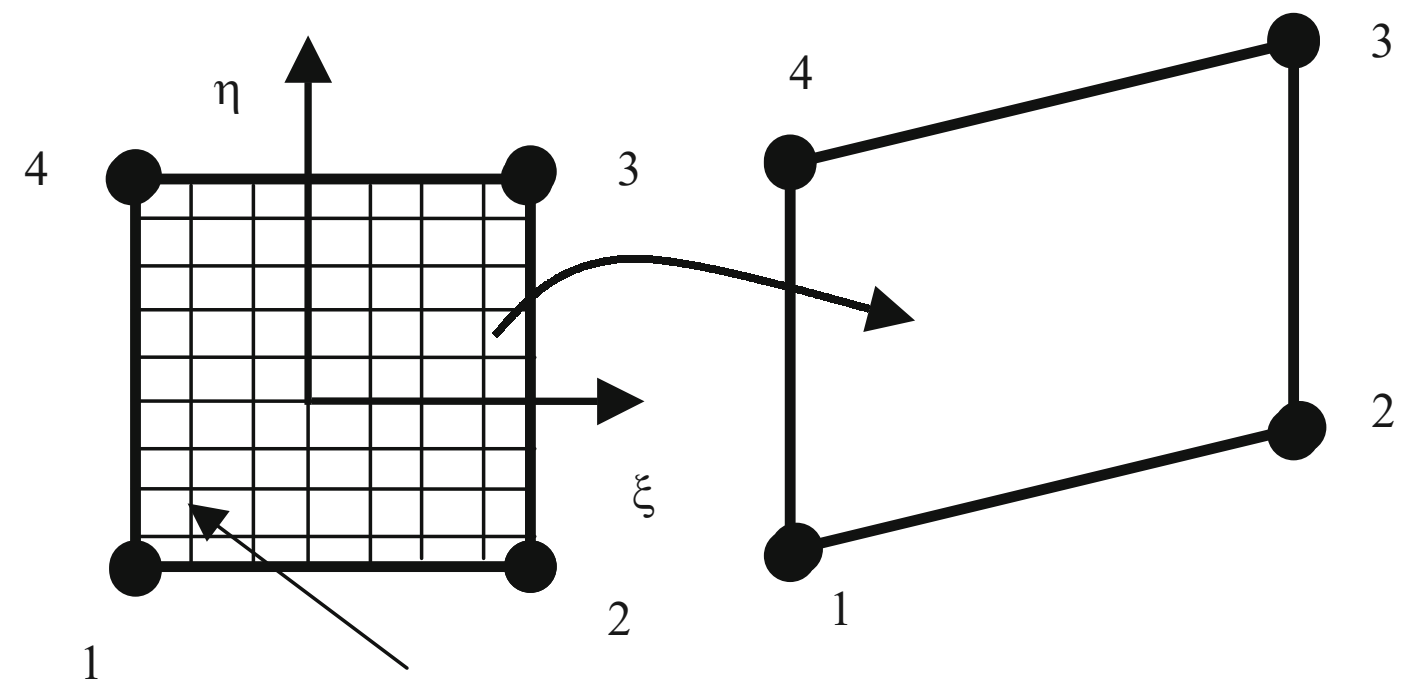

Mèches dans l'élément de référence

Figure 4. Elément finis à 4 nœuds spécifique aux tissés. Elément de référence, élément réel

Dans le présent élément, les interactions entre les mèches sens trame et chaîne ne sont pas pris en compte, en accord avec la constitution particulière du renfort très déséquilibré étudié pour lequel une direction est bien plus rigide que l'autre. Pour d'autres renforts l'effet du tissage et les interactions entre les mèches est important, le comportement en tension du renfort est alors identifié par des essais biaxiaux (Buet et al., 2001), (Boisse et al., 2001) et se caractérise par des surfaces de comportement. Associé aux coordonnées naturelles de l'élément $\xi, \eta$, on définit les vecteurs suivants :

$$
\mathbf{g}_{\alpha}=\frac{\partial \mathbf{x}}{\partial \xi_{\alpha}} \quad \mathrm{h}_{\alpha}=\frac{\mathbf{g}_{\alpha}}{\left\|\mathbf{g}_{\alpha}\right\|} \quad \mathbf{g}_{\alpha} \cdot \mathbf{g}^{\beta}=\delta_{\alpha}^{\beta} \quad \mathrm{h}_{\alpha} \cdot \mathrm{h}^{\beta}=\delta_{\alpha}^{\beta}
$$

Considérons dans un premier temps que les mèches sont orientées selon $\mathbf{g}_{1}$, le travail des efforts intérieurs s'écrit pour un élément :

$$
\mathrm{T}_{\text {int }}^{\mathrm{e}}(\boldsymbol{\eta})=\sum_{\text {mèche }} \int_{1} \varepsilon_{11} \mathrm{~T}^{11} \mathrm{ds}
$$


Les composantes du tenseur gradient, dans $\mathbf{h}_{\mathbf{1}}, \mathbf{h}_{\mathbf{2}}$, et $\mathbf{g}_{\mathbf{1}}, \mathbf{g}_{\mathbf{2}}$ sont sous la forme :

$$
\nabla^{\mathrm{s}} \boldsymbol{\eta}=\varepsilon_{\alpha \beta} \mathbf{h}^{\alpha} \otimes \mathbf{h}^{\beta}=\bar{\varepsilon}_{\alpha \beta} \mathbf{g}^{\alpha} \otimes \mathbf{g}^{\beta}
$$

Les composantes du tenseur déformation sont calculées à partir des fonctions d'interpolation sous la forme :

$$
\bar{\varepsilon}_{11}=\frac{\partial \boldsymbol{\eta}}{\partial \xi} \cdot \mathrm{g}_{1}=\frac{\partial \mathrm{N}^{\mathrm{k}}}{\partial \xi} \eta_{\mathrm{k}} \cdot \mathbf{g}_{1}=\mathrm{B}_{11 \mathrm{~s}} \eta_{\mathrm{s}}
$$

L'équation [10] devient alors :

$$
\mathrm{T}_{\text {int }}^{\mathrm{e}}(\boldsymbol{\eta})=\eta_{\mathrm{s}} \sum_{\text {mèche }} \int_{1} \frac{1}{\left\|\mathrm{~g}_{1}\right\|^{2}} \mathrm{~B}_{11 \mathrm{~s}} \mathrm{~T}^{11} \mathrm{ds}=\eta_{\mathrm{s}}\left(\mathrm{F}_{\text {int }}^{\mathrm{e}}\right)_{\mathrm{s}}
$$

Les quantités étant indépendantes de $\xi$, et par ailleurs $\mathrm{ds}=\left\|\mathbf{g}_{1}\right\| \mathrm{d} \xi$, les composantes du vecteur élémentaire des efforts intérieurs, s'écrivent alors pour des mèches dans le sens chaîne et trame orientées selon $\mathbf{g}_{1}, \mathbf{g}_{2}$ :

$$
\left(\mathrm{F}_{\text {int }}^{\mathrm{e}}\right)_{\mathrm{s}}=\sum_{\text {mèche chaine }} \frac{1}{\left\|\mathbf{g}_{1}\right\|} \mathrm{B}_{11 \mathrm{~s}} \mathrm{~T}^{11}+\sum_{\text {mèche trame }} \frac{1}{\left\|\mathbf{g}_{2}\right\|} \mathrm{B}_{22 \mathrm{~s}} \mathrm{~T}^{22}
$$

Puisque l'élément dispose de fonctions bilinéaires, la sommation sur le nombre de mèches dans l'équation [14] conduit à l'expression suivante :

$$
\left(\mathrm{F}_{\text {int }}^{\mathrm{e}}\right)_{\mathrm{s}}=\sum_{\mathrm{p}=1}^{2} \mathrm{~N}_{\mathrm{C}} \mathrm{B}_{11 \mathrm{~s}} \mathrm{~T}^{11} \frac{1}{\left\|\mathbf{g}_{1}\right\|}+\sum_{\mathrm{p}=1}^{2} \mathrm{~N}_{\mathrm{T}} \mathrm{B}_{22 \mathrm{~s}} \mathrm{~T}^{22} \frac{1}{\left\|\mathbf{g}_{2}\right\|}
$$

Les composantes du vecteur des efforts intérieurs peuvent être donc écrites de manière explicite comme la somme de 4 scalaires, ce qui fournit à l'élément une grande efficacité numérique. Implémenté dans un algorithme en dynamique explicite, il est utilisé de manière classique en intégration réduite avec le traitement spécifique des modes à énergie nulle (Belytschko et al., 1983). Les termes de tension, dans l'équation 15 , sont issus directement de la loi de comportement.

\section{Simulation de la mise en forme du renfort très déséquilibré}

Le procédé expérimental de mise en forme du renfort très déséquilibré, décrit au paragraphe 2 est simulé en utilisant les éléments à quatre nœuds décrits ci-dessus. Les maillages utilisés pour le renfort tissé, ainsi que pour le poinçon, la matrice et le 
serre-flan sont représentés figure 5. La déformée obtenue à la fin du procédé est représentée à la figure 6 , et est comparée à la forme expérimentale. L'allure générale est bien décrite par la simulation. Le point important porte sur les grandes différences des déformées dans les directions chaîne et trame.
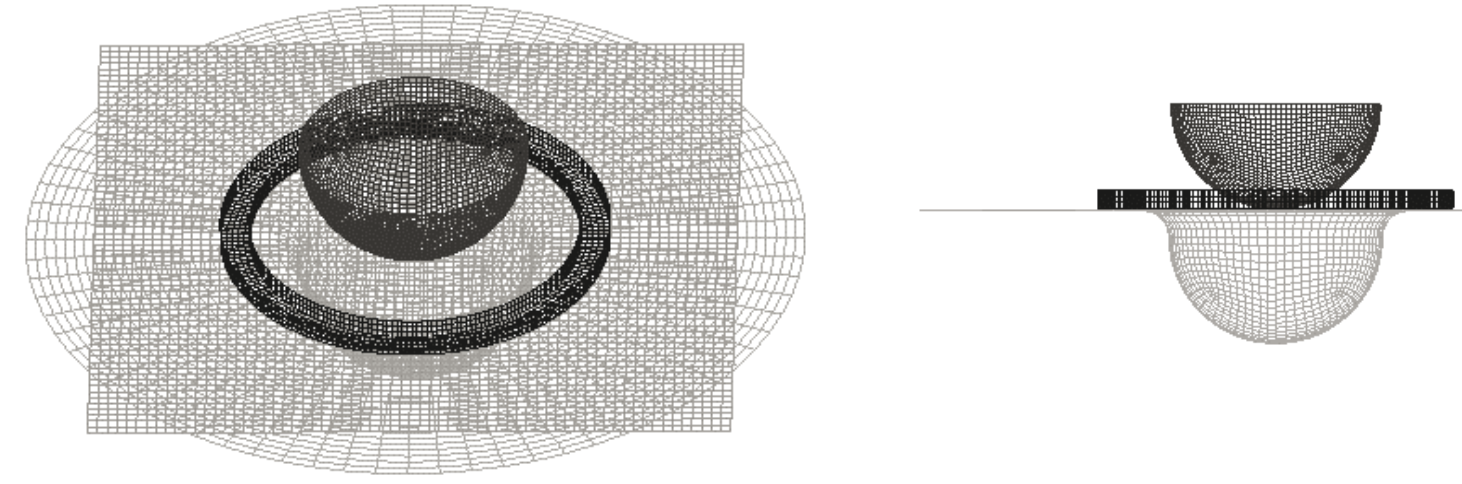

Figure 5. Maillages initiaux du renfort, matrice, poinçon et serre-flan

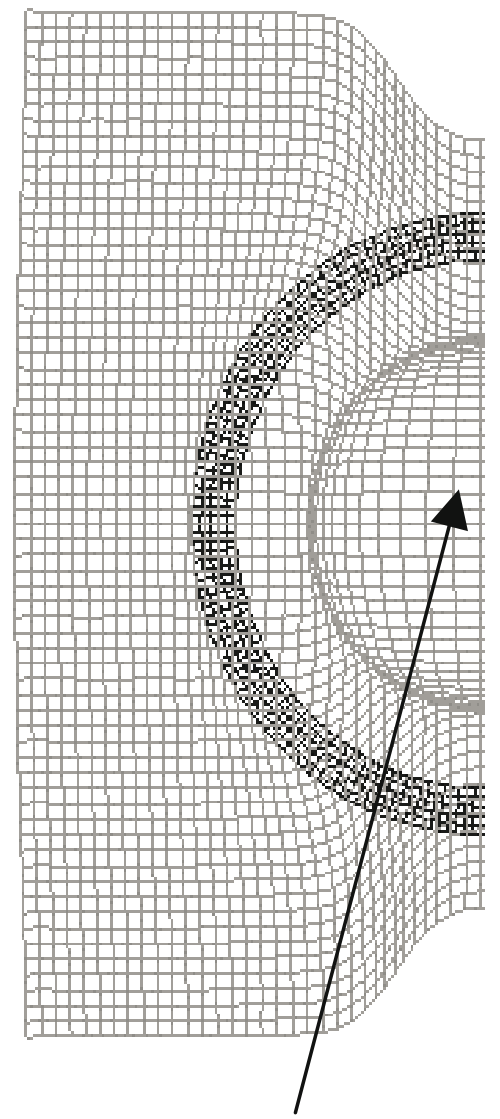

Ltrame/Lchaîne $=1,87$

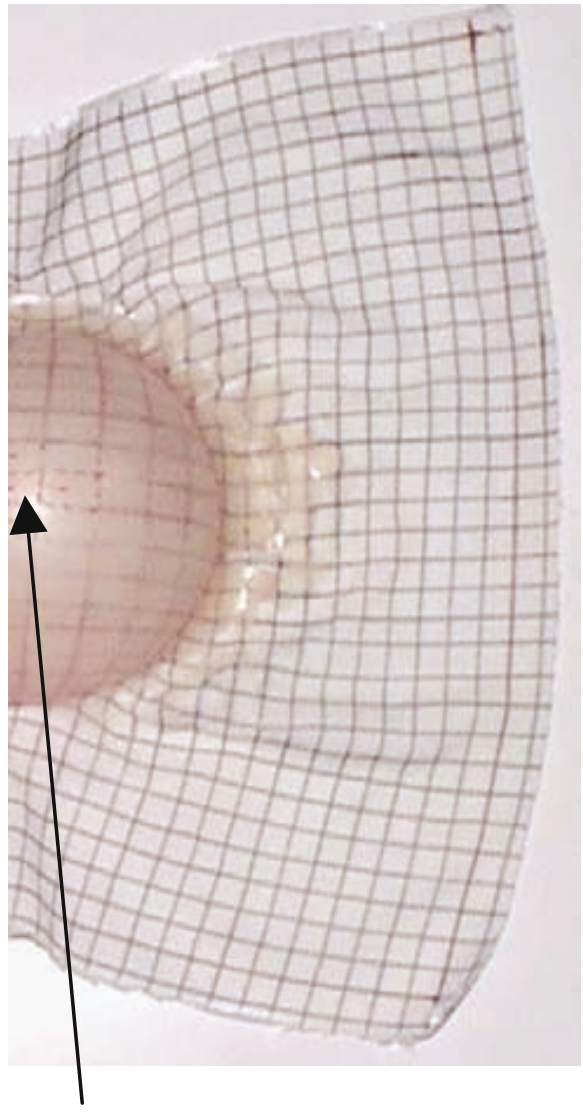

Ltrame $/$ Lchaîne $=1,8$

Figure 6. Comparaison des déformées : expérimentale (droite) et calculée (gauche) 
La direction trame est celle de plus faible rigidité (selon l'horizontale sur la figure 6). L'allongement dans cette direction est très important dans la zone centrale de par la présence du serre-flan. Les déplacements des points situés sur les arêtes dans la direction trame sont faibles. A l'opposé dans la direction chaîne (orientée verticalement), l'allongement est très faible et les points sur les arêtes dans la direction chaîne sont importants autour du centre de l'hémisphère. Au sommet de l'hémisphère, les carrés de la grille dessinée se déforment de manière importante et deviennent rectangulaires. Le rapport des longueurs des côtés (au sommet de l'hémisphère) dans les directions chaîne et trame sont précisés sur la figure 6 et il atteint 1,8. Par la simulation ce rapport est de 1,87, en bon accord avec ces résultats expérimentaux.

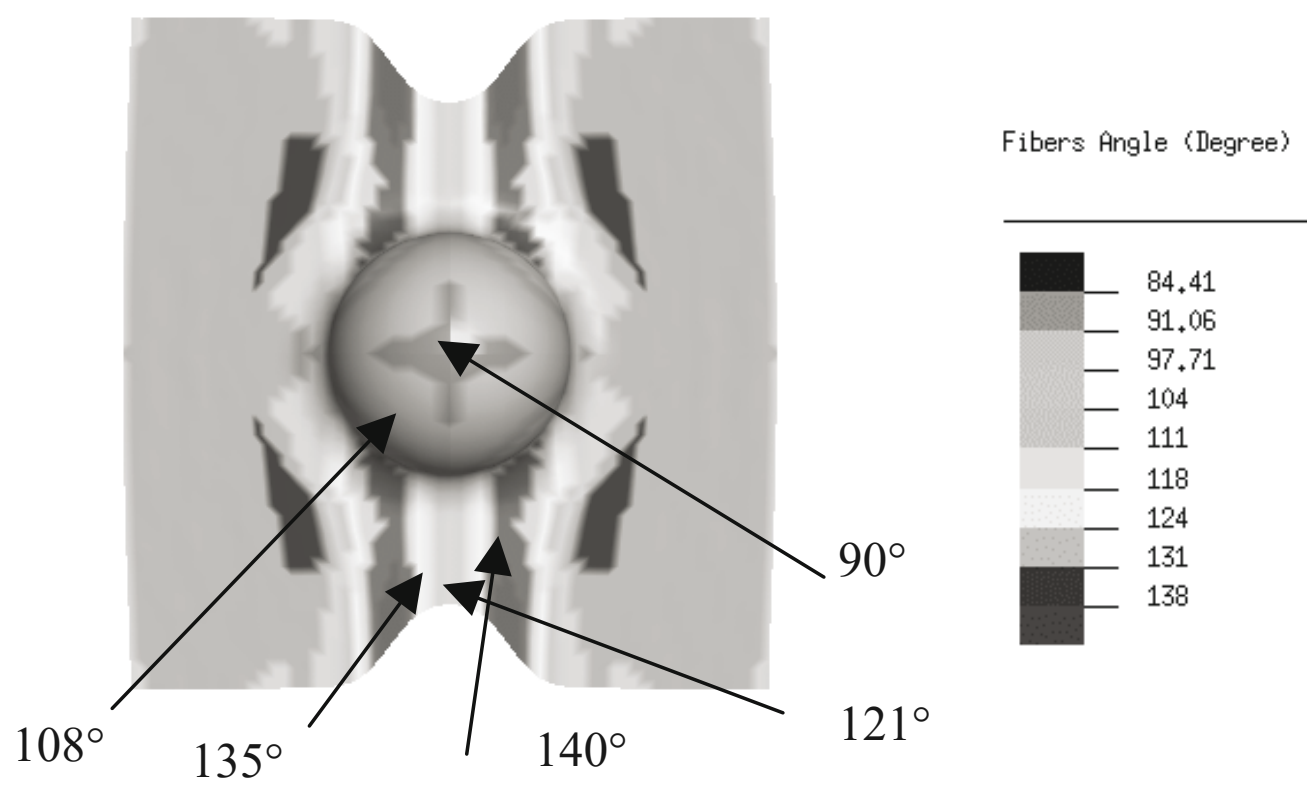

Figure 7. Angles entre les mèches chaînes et trame après la mise en forme

Notons que le même exemple traité avec des algorithmes géométriques de type drapage qui ne prennent pas en compte le comportement déséquilibré de ce renfort, conduirait à une déformée symétrique et à un rapport des longueurs égal à 1 en ce sommet.

La figure 7 représente les déformations de cisaillement dans le plan, c'est-à-dire les angles entre les mèches. Les zones de fortes déformations sont en bonne adéquation avec les résultats expérimentaux (Dumont, 2003) avant la formation des plis qui modifient les valeurs de ces variations angulaires. La répartition de ces angles n'est pas symétrique et dépend très fortement du déséquilibre du renfort. La figure 8 représente les tensions dans les mèches sens chaîne et trame. La connaissance de ces tensions est un aspect important par exemple pour réguler la pression imposée sur le serre-flan et éviter la rupture des mèches. Les tensions calculées particulièrement dans le sens trame sont importantes au sommet de 
l'hémisphère. La déformation calculée n'est pas très éloignée $(0,87)$ de la déformation à rupture qui est de 1,1 .

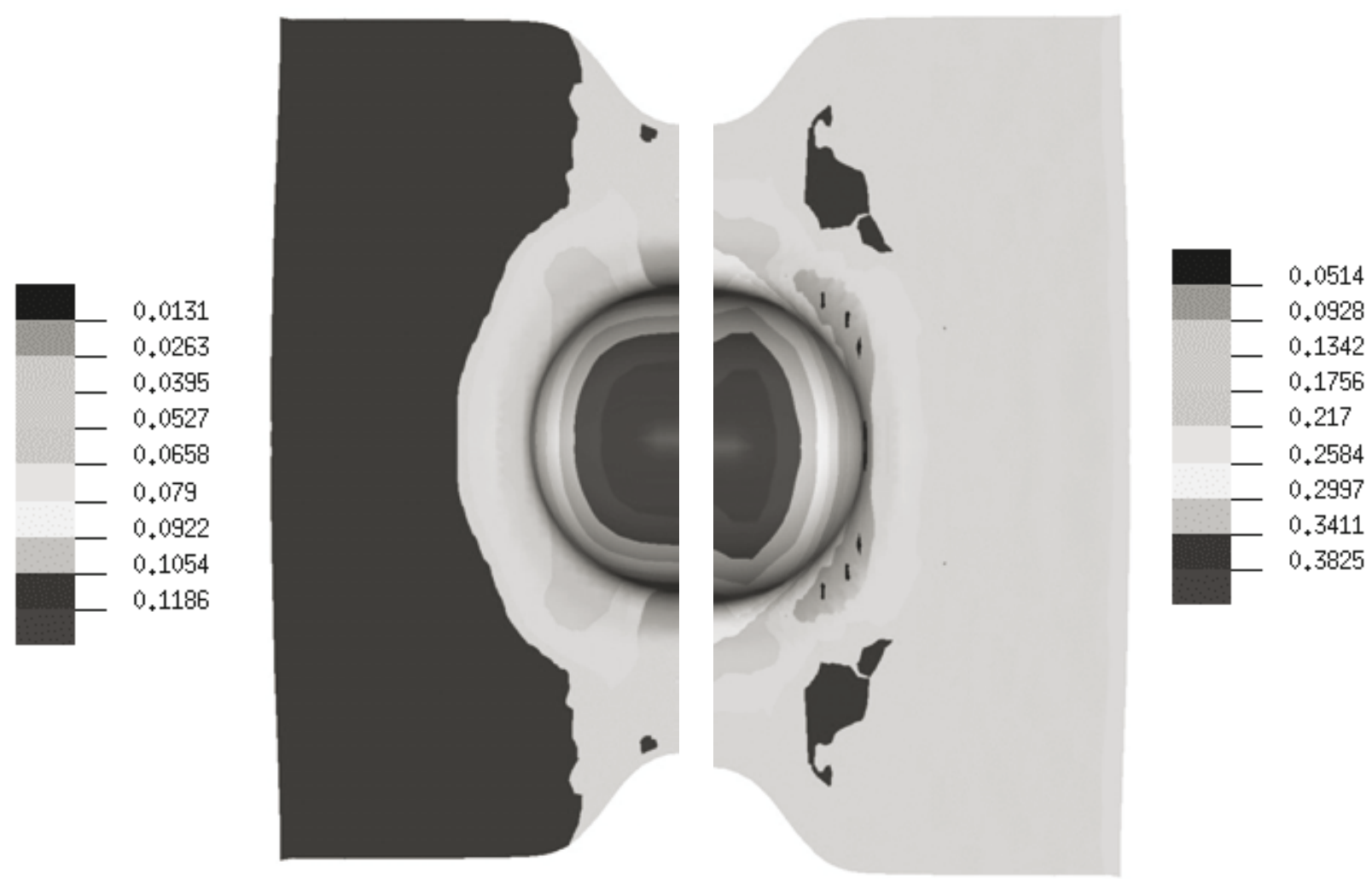

Figure 8. Tensions dans les mèches (en N/mèche), sens trame (à gauche), sens chaîne (à droite)

\section{Conclusion}

Une mise en forme expérimentale d'un renfort tissé très déséquilibré par un couple d'outils de géométrie hémisphérique a été réalisée. Elle conduit à des résultats très fortement non-symétriques. Ce résultat ne peut être reproduit par les algorithmes basés sur des approches géométriques de type drapage. Un élément fini spécifique aux tissés, basé sur les énergies de déformation en tension, a été développé et implémenté en dynamique explicite. Les résultats obtenus sont en bon accord avec les résultats expérimentaux, sur le calcul des déformations dans les mèches ou sur les variations d'angles entre les mèches qui constituent le principal mode de déformation des tissus « secs ». La formulation proposée est par ailleurs en bonne adéquation avec la physique des tissus puisque la loi de comportement intégrée est issue des essais de tensions biaxiaux. Cet exemple montre très clairement l'importance de la prise en compte des caractéristiques mécaniques du renfort sur la mise en forme. De la même manière les conditions aux limites, c'est-àdire les efforts sur les outils, influent considérablement sur le résultat de la mise en forme. L'approche par éléments finis permet de modéliser ces phénomènes. 
Par l'approche proposée, une formulation membranaire sans rigidité de flexion ou de cisaillement plan, il n'est pas possible de modéliser les plis qui sont présents dans la forme expérimentale. En revanche, il est possible, par les valeurs des angles entre les mèches, de détecter leur apparition. La modélisation de ces plis, par le développement d'un élément en version coque ou par l'ajout de rigidité de cisaillement plan, sont, entre autres, les perspectives prochaines des développements à réaliser.

\section{Bibliographie}

Belytschko T., "An overview of semidiscretisation and time integration procedures", Computation methods for transient analysis, ed. T. Belytschko \& T.J.R.Hughes, Elsevier Science, 1983, p. 1-65.

Belytschko T., Tsay C.S., "A stabilization procedure for the quadrilateral plate element with one point quadrature", International Journal for Numerical Methods in Engineering, vol. 19, 1983, p. 275-290.

Bickerton S., Simacek P., Guglielmi S.E., Advani S.G., "Investigation of draping and its effects on the mold filling process during manufacturing of a compound curved composite part", Composite Part A, vol. 28, 1997, p. 801-816.

Boisse P., Daniel J.L., Hivet G., Soulat D., "A simplified explicit approach for simulations of fibre fabric deformation during manufacturing preforms for R.T.M. process", International Journal of Forming Processes, vol.3, n³-4, 2001, p. 331-353.

Boisse P., Gasser A., Hivet G., "Analyses of fabric behaviour : determination of the biaxial tension-strain surfaces and their use in forming simulations", Composites Part A, vol. 3210, 2001, p. 1395-1414.

Buet K., Boisse P., "Experimental analysis and models for biaxial mechanical behaviour of composite woven reinforcements", Experimental Mechanics, vol. 41, $\mathrm{n}^{\circ}$ 3, 2001, p. $260-269$.

Carronnier D., Gay D., "Approche intégrée du RTM», Revue des composites et des matériaux avancés, vol. 6, Hermes, 1996.

Dumont F., Contribution à l'expérimentation et à la modélisation du comportement mécanique de renforts de composites, Thèse de doctorat, Université d'Orléans, 2003.

Hivet G., Launay J., Gasser A., Daniel J.L., Boisse P., "Mechanical Behaviour of Woven composites reinforcements while forming", Journal of thermoplastic Composite Materials, Vol. 15, n6, 2002, p. 545-555.

Long A.C., Rudd C.D., "A simulation of reinforcement deformation during the production of preforms for liquid moulding processes", I. Mech. E. J. Eng. Manuf., vol. 208, 1994, p. 269-278.

Rudd C.D., Turner M.R., Long A.C., Middleton V., "Tow placement studies for liquid composite moulding", Composites Part A, vol. 30-9, 1999, p. 1105-1121. 
Van Der Ween F., "Algorithms for draping fabrics on doubly curved surfaces", International Journal of Numerical Method in Engineering, vol. 31, 1991, p. 1414-1426.

Wang J., Paton R., Paye J.R., "The draping of woven fabric preforms and prepregs for production of polymer composite components", Composites A, vol. 30, 1999, p. 757-765.

Zienkiewicz O., Taylor R., The finite element method, vol. 1, Ed. Mc GrawHill, 1989. 